\title{
Welche Zukunft hat die spinale (Neuro)chirurgie in der Schweiz?
}

\section{Raoul Heilbronner}

Past-president

Schweizerische Gesellschaft

für Neurochirurgie,

ehemaliger Sekretär der

Schweizerischen Gesellschaft

für spinale Chirurgie

\section{Wirbelsäulenchirurgie und kranielle Neurochirurgie}

Neurochirurgie ist in den meisten neurochirurgischen Abteilungen oder Kliniken der Schweiz primär einmal Wirbelsäulenchirurgie. Der Anteil spinaler Chirurgie in den anerkannten neurochirurgischen Weiterbildungsstätten beträgt bis zu $100 \%$. In den grossen Zentren beträgt der Anteil der spinalen Eingriffe 30-70\% aller Operationen. Eine Ausnahme stellt die neurochirurgische Universitätsklinik in Zürich dar, die sich fast ausschliesslich der kraniellen Neurochirurgie widmet.

Die häufigsten Eingriffe an der Wirbelsäule in den neurochirurgischen Abteilungen haben die Entlastung der neuralen Strukturen zum Ziel. Sie werden heute meist mikrochirurgisch durchgeführt. Beispiele sind lumbale und zervikale Diskushernien mit Neurokompression, degenerative Stenosen mit Kompression der Cauda equina oder des Halsmarkes. Weitaus seltener sind intradurale Pathologien. Um all diese Eingriffe zu erlernen, ist eine mehrjährige neurologisch-mikrochirurgische Weiterbildung unumgänglich. Dies ist bislang eine spezifisch neurochirurgische Domäne. Eine zunehmende Bedeutung haben stabilisierende Massnahmen an der Wirbelsäule erhalten.

\section{«Neurochirurgische Einheiten ohne spinale Chirurgie} wären wohl nur noch an sechs bis sieben Spitälern in der Schweiz möglich»

Korrespondenz:

Dr. med. Raoul Heilbronner Klinik für Neurochirurgie Kantonsspital St. Gallen

CH-9007 St. Gallen

Tel. 0714941674

Fax 0714942883

raoul.heilbronner@kssg.ch
Der Stellenwert der spinalen Chirurgie in der Neurochirurgie spiegelt sich im FMH-Weiterbildungsprogramm für Neurochirurgie wieder. Letzteres verlangt Wirbelsäuleneingriffe für mehr als die Hälfte des geforderten OP-Katalogs (135 Eingriffe von einem Total von 266). Die Weiterbildungsordnung der Orthopädie ist hingegen nicht schwerpunktmässig spinal ausgerichtet. Jeder Neurochirurge FMH ist somit primär ein spinaler Chirurge. Noch deutlicher zeigt sich dies in der Privatpraxis, wo der Anteil spinaler Eingriffe bei Neurochirurgen über $90 \%$ beträgt.

Ausserhalb der Schweiz bietet sich ein buntes Bild. Es gibt Länder, in denen die spinale Chirurgie (Rückenmarkstumore usw. ausgenommen) weitgehend von Orthopäden, aber auch solche, in denen sie praktisch ausschliesslich von Neurochirurgen betrieben wird.

Noch bis in die Neunzigerjahre hinein haben die grossen neurochirurgischen Weiterbildungszentren in der Schweiz der Instrumentation an der Wirbelsäule

\section{Quel est l'avenir de la (neuro-) chirurgie spinale en Suisse?}

La neurochirurgie est majoritairement la chirurgie de la colonne vertébrale. Mais la chirurgie de la colonne vertébrale, c'est aussi l'orthopédie. Le programme de formation postgraduée de la FMH pour la neurochirurgie exige plus d'opérations spinales que crâniennes et il est le seul à exiger, de manière sélective, des interventions sur la colonne vertébrale. Néanmoins, les orthopédistes de Suisse ont mis sur pied de très bons services de chirurgie spinale aptes à concurrencer les services de neurochirurgie. On envisage de créer une association des services de neurochirurgie et de chirurgie orthopédique spinale pour des raisons professionnelles et économiques. Mais si un tel plan est réalisé à la hâte, cette précipitation menacera non seulement le mandat de formation confié aux établissements de formation postgraduée en neurochirurgie et chirurgie orthopédique mais aussi l'existence des unités de neurochirurgie plus petites, car celles-ci, sans la chirurgie spinale, ne pourront plus guère être gérées que dans les grands hôpitaux pour des raisons économiques.

Les sociétés suisses d'orthopédie, de neurochirurgie et de chirurgie spinale tiennent (et elles font bien d'agir ainsi) à concevoir avec circonspection - et dans le maintien d'une orthopédie et d'une neurochirurgie de haute qualité - des projets susceptibles de garantir une chirurgie de la colonne vertébrale également de haute qualité en Suisse.

nicht den ihr zustehenden Stellenwert entgegengebracht. Spinal engagierte Neurochirurgen wie Benini oder Markwalder waren eine Ausnahme. Die Schweizer Wirbelsäulenorthopäden pflegten dieses Gebiet mit Nachdruck. Dick, Morscher, Magerl und andere sind diesbezüglich bestens bekannt. Ganz im Gegensatz zu den Wirbelsäulenorthopäden, die in der Schweiz auf eine grosse Tradition mit weltberühmten Exponenten wie zum Beispiel Dick, Morscher und Magerl verwei- 
sen können. Heute ist eine hochstehende Wirbelsäulenchirurgie ohne diese wirbelsäulenorthopädischen Beiträge unvorstellbar. Engagierte Orthopäden haben in der Schweiz sehr gute spinale Abteilungen auf die Beine gestellt, die direkt mit den neurochirurgischen konkurrieren. Das grosse Volumen der degenerativen Erkrankungen wird von beiden Fachrichtungen abgedeckt. Es verbleiben jedoch spezifisch orthopädische oder neurochirurgische Teilgebiete, wie zum Beispiel die Skoliosenchirurgie, respektive die Chirurgie der intraduralen Pathologien.

Mit einem gewissen Recht fragen sich die administrativen Leitungen der grossen Weiterbildungsspitäler, wieso man gleich zwei Abteilungen betreiben soll, die sich der Chirurgie an der Wirbelsäule widmen, und ob man diese Abteilungen wohl nicht besser aus ihren Mutterkliniken ausgliedern und zusammenlegen sollte.

Die flächendeckende Umsetzung solcher Pläne hätte eine massgebliche Veränderung der neurochirurgischen Landschaft in der Schweiz zur Folge. Neurochirurgische Einheiten ohne spinale Chirurgie wären wohl nur noch an sechs bis sieben Spitälern in der Schweiz möglich, da ohne spinale Chirurgie die nötige Arbeitslast fehlte, um einen Betrieb 24 Stunden täglich und an 365 Tagen pro Jahr personell und apparativ wirtschaftlich betreiben zu können. Also müsste der Notfallauftrag in Spitälern mit kleineren Neurochirurgien durch den Wegfall dieser Abteilungen neu definiert werden.

Man bedenke, dass ohne Neurochirurgie eine umfassende Versorgung von Patienten mit Schädel-HirnTraumen oder das Betreiben von Stroke-Units nicht möglich ist!

Es ist keine Frage: Es steht die Überführung der gesamten spinalen Chirurgie in klinisch und operativ getrennte, personell und räumlich selbstständige Einheiten und unter einer von der Neurochirurgie und Orthopädie weitgehend losgelösten Leitung zur Diskussion.

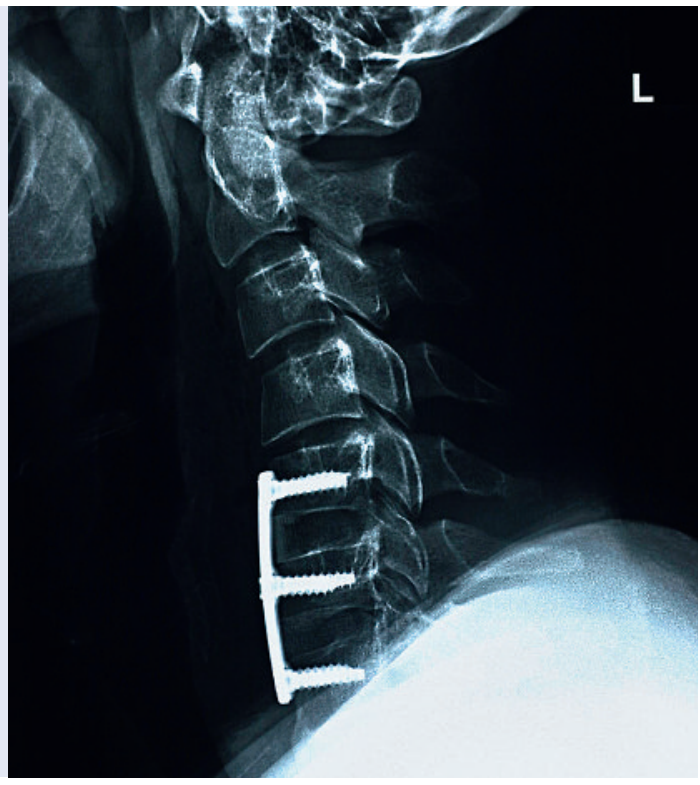

Eine überstürzte Umsetzung solcher Pläne hätte eine vitale Gefährdung des Weiterbildungsauftrages der neurochirurgischen und orthopädischen Kliniken zur Folge. Neurochirurgische und orthopädische A-Kliniken könnten den A-Status verlieren, womit wohl auch ein Abstieg der örtlichen spinalen Chirurgie in die Zweitklassigkeit drohte. Es müssen also nicht nur die verbindlichen FMH-Weiterbildungsprogramme berücksichtigt werden, sondern auch, dass für eine fachlich vollständige Wirbelsäulenchirurgie neurobzw. mikrochirurgische und orthopädische Fertigkeiten von essentieller Bedeutung sind.

\section{Vermehrte Zusammenarbeit}

Eine Lösung, wie man Synergien bündelt, ohne die fachspezifischen Stärken zu schwächen, dürfte die Bildung von interdisziplinären Abteilungen darstellen, die unter einer paritätischen Leitung der Orthopädie und Neurochirurgie stehen. Ziel wäre ein Schliessen der aktuellen Weiterbildungs-, Versorgungs- und Kompetenzlücken und die Schaffung klarer Strukturen für die Ärzteschaft wie auch für die Patienten. Spinal tätige Schweizer Neurochirurgen und Orthopäden sind sich einig, dass für eine hochstehende spinale Chirurgie spezifische Fertigkeiten aus beiden Fachgebieten nötig sind. Leider arbeiten Neurochirurgen und Orthopäden aktuell noch wenig zusammen. Ansätze, dies zu ändern, sind an einigen Häusern glücklicherweise vorhanden.

Sowohl die Schweizerische Gesellschaft für spinale Chirurgie als auch die Schweizerische Gesellschaft für Neurochirurgie haben Arbeitsgruppen gebildet, die sich mit der Schaffung eines Fähigkeitsausweises oder eines Schwerpunktes in spinaler Chirurgie befassen. Zudem werden sich die Vorstände der SGN und SGO noch diesen Herbst an einen Tisch setzen und dieses Thema diskutieren. Es soll eine hochstehende spinale Chirurgie in der Schweiz gesichert werden. Dies wird eine wesentlich engere Zusammenarbeit von Neurochirurgen und Orthopäden verlangen als bis anhin, was ja auch gewünscht ist. Vernünftigerweise werden diese Überlegungen mit Blick auf die Entwicklung der Ausbildungsgänge in spinaler Chirurgie in Europa (und auch weltweit) angestellt.

Der Autor unterstützt dieses Vorgehen ausdrücklich, und zwar mit dem Argument, dass eine hochstehende Wirbelsäulenchirurgie mit der Sicherung einer möglichst guten Ausbildung unserer zukünftigen Wirbelsäulenchirurgen steht und fällt. Die zukünftige Gestaltung der Wirbelsäuleneinheiten in den Spitälern soll sich nach der Qualität richten und darf nicht von örtlichen Machtgefügen und rein wirtschaftlichen oder marketingtechnischen Faktoren abhängig sein.

Die Schweizer Neurochirurgen und Wirbelsäulenorthopäden werden in den nächsten Jahren im Interesse einer hochklassigen spinalen Chirurgie und auch aus Gründen der Ressourcen vermehrt zusammenarbeiten müssen. Darin primär eine Bedrohung der fachlichen Eigenständigkeit zu sehen, ist engstirnig und verdeckt die Aussicht auf die konstruktiven Synergien, die aus den beiden Fachbereichen erwachsen.
Neurochirurgie ist mehrheitlich Wirbelsäulenchirurgie. 